\title{
Inhibitory Effects of 4-n-Butylresorcinol on Tyrosinase Activity and Melanin Synthesis
}

\author{
Dong-Seok Kim,${ }^{a, b}$ So-Young KIM, ${ }^{a}$ Seo-Hyoung Park, ${ }^{a}$ Yeong-Gon ChoI, ${ }^{a}$ Sun-Bang Kwon, ${ }^{a}$ \\ Myo-Kyoung KIM, ${ }^{c}$ Jung-Im NA, ${ }^{a}$ Sang-Woong Youn, ${ }^{a}$ and Kyoung-Chan PARK ${ }^{*, a}$ \\ ${ }^{a}$ Department of Dermatology, Seoul National University College of Medicine; ${ }^{b}$ Research Division for Human Life \\ Sciences, Seoul National University; 28 Yongon-Dong, Chongno-Gu, Seoul 110-744, Korea: and ${ }^{c}$ Pharmacy Practice, \\ Thomas J. Long School of Pharmacy, University of the Pacific; Stockton, CA, U.S.A. \\ Received July 20, 2005; acceepted September 10, 2005
}

In this study, we investigated the effects of 4-n-butylresorcinol on melanogenesis in a spontaneously immortalized mouse melanocyte cell line, Mel-Ab. Our results show that 4 - $n$-butylresorcinol significantly inhibits melanin synthesis in a concentration-dependent manner. In addition, it was also found to inhibit the activity of tyrosinase, the rate-limiting melanogenic enzyme. Several reports have indicated that the activation of extracellular signal-regulated kinase (ERK) or of Akt reduces melanin synthesis via microphthalmia-associated transcription factor (MITF) down-regulation. Accordingly, we examined the effects of 4-n-butylresorcinol on the ERK and Akt signaling pathways. 4-n-Butylresorcinol did not induce ERK, Akt activation, or MITF degradation, and had no effect on cAMP response element binding protein (CREB) phosphorylation, which stimulates MITF expression. In contrast, 4-n-butylresorcinol strongly reduced tyrosinase activity in a cell-free system. Moreover, 4- $n$ butylresorcinol showed an additive effect in combination with hinokitiol, which reduces MITF expression. These results show that the hypopigmentary effect of 4-n-butylresorcinol results from its direct inhibition of tyrosinase.

Key words 4- $n$-butylresorcinol; melanogenesis; tyrosinase; microphthalmia-associated transcription factor (MITF)

The synthesis and distribution of melanin contributes to skin and hair color. However, increased levels of epidermal melanin synthesis can darken the skin and induce cosmetic problems. In mammalian melanocytes, melanins are synthesized within melanosomes that contain tyrosinase, which plays a key role in melanogenesis, as it catalyzes the ratelimiting reaction of the melanogenic process. ${ }^{1-3)}$ Accordingly, melanin production is mainly controlled by the expression and activation of tyrosinase. ${ }^{4)}$ Certain resorcinol derivatives have been known to inhibit tyrosinase activity. ${ }^{5)}$ Moreover, it has been reported that compounds with a 4-substituted resorcinol skeleton show potent tyrosinase inhibitory effects. ${ }^{6)}$ However, the effects and mechanism of 4- $n$-butylresorcinol on melanogenesis have not been clearly elucidated.

Microphthalmia-associated transcription factor (MITF) strongly stimulates tyrosinase expression, which indicates that it is an important regulator of melanogenesis. ${ }^{7-10)}$ Moreover, in humans, mutations in the MITF gene cause Waardenburg Syndrome type IIA, which manifests as an abnormal pigmentation of the skin and hair. ${ }^{11-13)}$

In recent reports, the inhibition of the extracellular signalregulated kinase (ERK) pathway was found to induce hyperpigmentation, suggesting that the ERK signaling pathway is involved in melanogenesis. ${ }^{14-16)}$ Furthermore, a $90 \mathrm{kDa}$ ribosomal S6 kinase (RSK-1) was found to phosphorylate MITF, and thus to lead to its degradation. ${ }^{17)}$ In addition, the activation of the Akt signaling pathway was also implicated in the inhibition of melanogenesis. ${ }^{18)}$ On the other hand, cAMP response element binding protein (CREB) phosphorylation was found to induce the expression of MITF and to increase melanin synthesis. ${ }^{9,19,20)}$

In this study, we investigated the effects of 4-n-butylresorcinol on melanin synthesis and tyrosinase activity in a spontaneously immortalized mouse melanocyte cell line, Mel-Ab. In addition, we examined its effects on several signaling pathways related to melanogenesis.

* To whom correspondence should be addressed. e-mail: gcpark@snu.ac.kr

\section{MATERIALS AND METHODS}

Materials 4- $n$-Butylresorcinol was synthesized by SK chemicals (Suwon, Korea). Hinokitiol, 12-O-tetradecanoylphorbol-13-acetate (TPA), cholera toxin (CT), synthetic melanin, L-DOPA, and mushroom tyrosinase were obtained from Sigma-Aldrich Co. (St. Louis, MO, U.S.A.). Antibodies recognizing phospho-specific CREB (Ser133, \#9196), phospho-specific MEK (Ser217/221, \#9121), phospho-specific ERK1/2 (Thr202/Tyr204, \#9101S), phospho-specific RSK-1 (Thr359/Ser363, \#9344S), and phospho-specific Akt (Ser473, \#9271S) were purchased from Cell Signaling Technology. Microphthalmia Ab-1 (C5, MS-771-P0) was purchased from NeoMarkers (Fremont, CA, U.S.A.), and actin (I-19) antibody from Santa Cruz Biotechnology, Inc. (Santa Cruz, CA, U.S.A.).

Cell Cultures Mel-Ab cell line is a mouse-derived spontaneously immortalized melanocyte cell line that produces large amounts of melanin. ${ }^{21)}$ Mel-Ab cells were incubated in DMEM supplemented with $10 \%$ fetal bovine serum (FBS), $100 \mathrm{~nm}$ TPA, $1 \mathrm{~nm} \mathrm{CT,} 50 \mu \mathrm{g} / \mathrm{ml}$ streptomycin, and $50 \mathrm{U} / \mathrm{ml}$ penicillin at $37^{\circ} \mathrm{C}$ in $5 \% \mathrm{CO}_{2}$.

Cell Viability Assay Cell viability was determined using a crystal violet assay. ${ }^{21)}$ After incubating cells with test substances for $24 \mathrm{~h}$, the medium was removed and stained with $0.1 \%$ crystal violet in $10 \%$ ethanol for $5 \mathrm{~min}$ at room temperature and then rinsed four times with distilled water. Crystal violet retained by adherent cells was extracted with $95 \%$ ethanol, and absorbance was determined at $590 \mathrm{~nm}$ using an ELISA reader (TECAN, Salzburg, Austria).

Measurement of Melanin Contents and Microscopy Melanin contents were measured as described previously, ${ }^{22}$ ) with a slight modification. Briefly, cells were treated with test substances in DMEM containing 2\% FBS for $4 \mathrm{~d}$. Cell pellets were then dissolved in $1 \mathrm{ml}$ of $1 \mathrm{~N} \mathrm{NaOH}$ at $100^{\circ} \mathrm{C}$ for $30 \mathrm{~min}$ and centrifuged for $20 \mathrm{~min}$ at $16000 \times \mathbf{g}$. Optical den- 
sities (OD) of the supernatants were measured at $400 \mathrm{~nm}$ using an ELISA reader. A standard synthetic melanin curve ( 0 to $300 \mu \mathrm{g} / \mathrm{ml}$ ) was prepared in triplicate for each experiment. Before determining melanin contents, cells were observed under a phase contrast microscope (Olympus Optical Co., Tokyo, Japan) and photographed using a CoolSNAP digital video camera system (Roper Scientific, Inc., Tucson, AZ, U.S.A.), which was supported by RS Image software (Roper Scientific, Inc., Tucson, AZ, U.S.A.).

Tyrosinase Activity Tyrosinase activity was determined as previously described ${ }^{23)}$ with slight modification. Briefly, $\mathrm{Mel}-\mathrm{Ab}$ cells were cultured in $60 \mathrm{~mm}$ dishes. After incubating with test substances in DMEM containing 2\% FBS for $4 \mathrm{~d}$, the cells were washed with ice-cold PBS and lysed with phosphate buffer ( $\mathrm{pH}$ 6.8) containing 1\% Triton X-100. The cells were then disrupted by freeze-thawing, and lysates were clarified by centrifuging at $10000 \times \boldsymbol{g}$ for $5 \mathrm{~min}$. After quantifying protein levels and adjusting concentrations with lysis buffer, $90 \mu \mathrm{l}$ of each lysate, was placed in a well of a 96-well plate, and $10 \mu \mathrm{l}$ of $10 \mathrm{~mm}$ L-DOPA was then added. Control wells contained $90 \mu \mathrm{l}$ of lysis buffer and $10 \mu \mathrm{l}$ of $10 \mathrm{~mm} \mathrm{~L}-$ DOPA. After incubation at $37^{\circ} \mathrm{C}$, absorbance was measured every $10 \mathrm{~min}$ for at least $1 \mathrm{~h}$ at $475 \mathrm{~nm}$ using an ELISA reader. A cell-free assay system was used to test for the direct effects on tyrosinase activity. Seventy microliters of phosphate buffer containing the various test substances were mixed either with $20 \mu \mathrm{l}$ of $10 \mu \mathrm{g} / \mathrm{ml}$ mushroom tyrosinase or with $20 \mu \mathrm{l}$ of human tyrosinase (as $20 \mu \mathrm{g}$ of total protein) extracted from primary cultured human melanocytes, and $10 \mu \mathrm{l}$ of $10 \mathrm{~mm}$ L-DOPA was added to each well. Following incubation at $37^{\circ} \mathrm{C}$, absorbance was measured at $475 \mathrm{~nm}$.

Western Blot Analysis Cells were lysed in cell lysis buffer [62.5 mm Tris- $\mathrm{HCl}$ (pH 6.8), 2\% SDS, 5\% $\beta$-mercaptoethanol, $2 \mathrm{~mm}$ phenylmethylsulfonyl fluoride, protease inhibitors (Complete ${ }^{\mathrm{TM}}$, Roche, Mannheim, Germany), $1 \mathrm{~mm}$ $\mathrm{Na}_{3} \mathrm{VO}_{4}, 50 \mathrm{~mm} \mathrm{NaF}$, and $10 \mathrm{~mm}$ EDTA]. Ten micrograms of protein per lane was separated by SDS-polyacrylamide gel electrophoresis and blotted onto PVDF membranes, which were saturated with $5 \%$ dried milk in Tris-buffered saline containing $0.4 \%$ Tween 20 . Blots were incubated with the appropriate primary antibodies at a dilution of $1: 1000$, and then further incubated with horseradish peroxidase-conjugated secondary antibody. Bound antibodies were detected using an enhanced chemiluminescence plus kit (Amersham International, Little Chalfont, U.K.).

Statistics Differences between results were assessed for significance using the Student's $t$-test. $p$ values of $<0.01$ were taken to be significant.

\section{RESULTS}

Effects of 4-n-Butylresorcinol on Melanin Synthesis in Mel-Ab Cells Structurally, 4- $n$-butylresorcinol is a phenol derivative with a hydroxyl group at the meta-position and a butyl group at the para-position (Fig. 1). To examine whether 4-n-butylresorcinol has cytotoxic effects, we treated Mel-Ab cells with 4- $n$-butylresorcinol at various concentrations; cell viability was determined by using the crystal violet assay. Figure 2 shows that $4-n$-butylresorcinol is not cytotoxic to $\mathrm{Mel}-\mathrm{Ab}$ cells in the concentration range of $1-100 \mu \mathrm{M}$. To investigate the effect of 4- $n$-butylresorcinol, Mel-Ab cells were

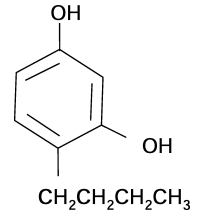

Fig. 1. The Structure of 4- $n$-Butylresorcinol

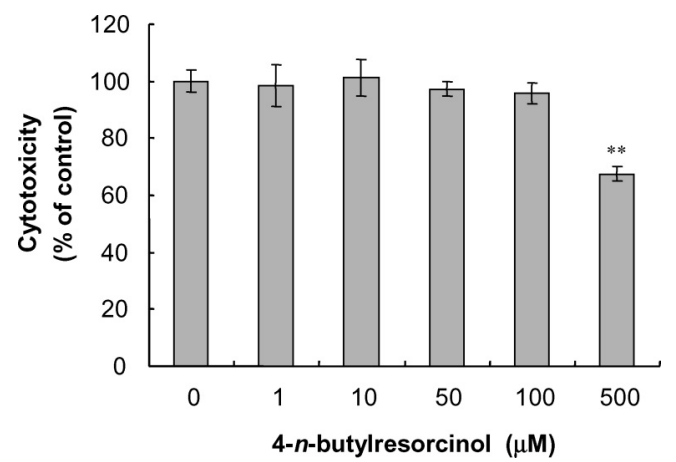

Fig. 2. Effects of 4-n-Butylresorcinol on Mel-Ab Cell Viability

Cells were serum-starved for $24 \mathrm{~h}$ and $4-n$-butylresorcinol was treated in serum-free media at $1-500 \mu \mathrm{M}$ for $24 \mathrm{~h}$. Cell viabilities were determined by crystal violet assay. Each determination was made in triplicate and data shown are means \pm S.D. $* * p<0.01$ compared to control.

exposed to $0.1-100 \mu \mathrm{M} 4-n$-butylresorcinol for $4 \mathrm{~d}$ and then photographed under a phase contrast microscope (Fig. 3A). The results obtained showed that the 4- $n$-butylresorcinoltreated cells were markedly less pigmented. We also measured cellular melanin contents after 4- $n$-butylresorcinol treatment. As shown in Fig. 3B, the melanin contents of cells were significantly down-regulated by $4-n$-butylresorcinol, at concentrations higher than $10 \mu \mathrm{M}$.

4-n-Butylresorcinol Does Not Influence Signaling Pathways Related to Melanogenesis It is known that ERK activation induces MITF degradation, which reduces melanogenesis. $^{17,24)}$ Therefore, we examined whether 4- $n$-butylresorcinol influences the ERK signaling pathway. As shown in Fig. 4, 4- $n$-butylresorcinol neither activated ERK nor MEK (the upstream kinase of ERK) nor RSK-1 (the downstream kinase of ERK), and thus did not trigger a MITF mobility shift or degradation, which can be induced by ERK phosphorylation. ${ }^{15,17)}$ Additionally, the phosphorylation levels of CREB and Akt were examined after 4- $n$-butylresorcinol treatment, but they were unaltered.

4-n-Butylresorcinol Inhibits Tyrosinase Directly The direct effects of $4-n$-butylresorcinol on tyrosinase activity were also investigated using human tyrosinase. 4- $n$-Butylresorcinol showed a strong inhibitory effect on tyrosinase activity in a concentration-dependent manner (Fig. 5). We repeated the experiment with mushroom tyrosinase and obtained the same result (data not shown). Our results indicate that the inhibitory effect of $4-n$-butylresorcinol on melanogenesis is due to its direct inhibition of tyrosinase. To confirm this direct effect of 4- $n$-butylresorcinol, we used 4- $n$ butylresorcinol in combination with hinokitiol, which reduces MITF levels. As shown in Fig. 6, the combination of 4- $n$-butylresorcinol and hinokitiol acted in an additive manner, thus indicating that the strong tyrosinase inhibitory activity of 4- $n$-butylresorcinol works in combination with the reduced level of MITF induced by hinokitiol. 
A

Control
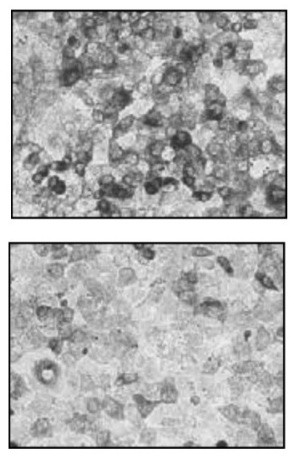

$10 \mu \mathrm{M}$
$0.1 \mu \mathrm{M}$
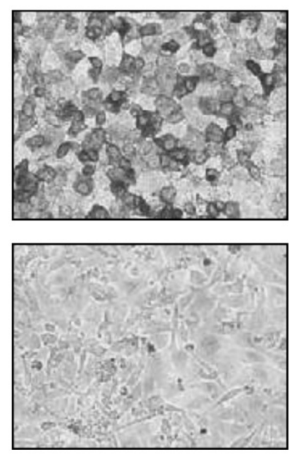

$50 \mu \mathrm{M}$
$1 \mu \mathrm{M}$
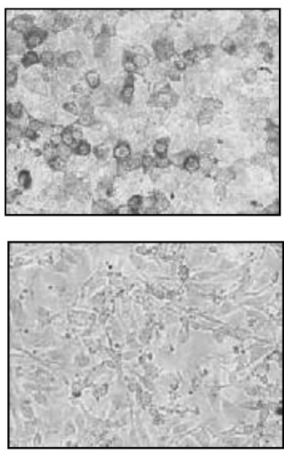

$100 \mu \mathrm{M}$
B

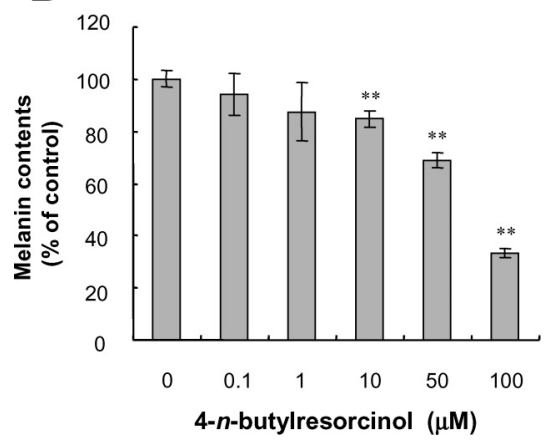

C

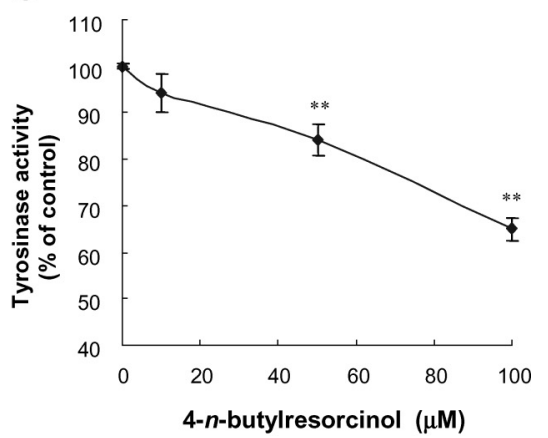

Fig. 3. Effects of 4- $n$-Butylresorcinol on Melanin Synthesis in Mel-Ab Cells

Cells were cultured with $1-100 \mu \mathrm{M}$ of $4-n$-butylresorcinol for $4 \mathrm{~d}$. Phase contrast photomicrographs (A) were taken using a digital video camera, as described in Materials and Methods, and melanin contents (B) and tyrosinase activity (C) were measured as described in Materials and Methods. The results shown are the averages of triplicate experiments \pm S.D. $* * p<0.01$ compared to the untreated control.

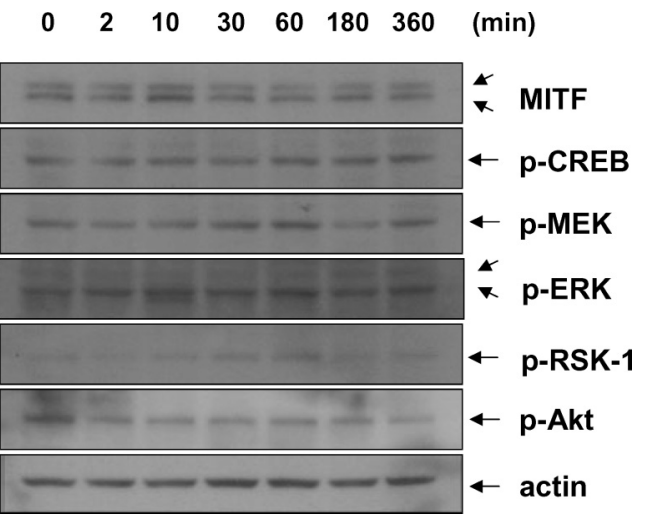

Fig. 4. Effects of 4- $n$-Butylresorcinol on Signaling Pathways in Mel-Ab Cells

After $24 \mathrm{~h}$ of serum starvation, Mel-Ab cells were treated with 4- $n$-butylresorcinol at the times indicated. Whole cell lysates were then subjected to Western-blot analysis using antibodies against MITF, phospho-specific CREB (p-CREB), phospho-specific MEK (p-MEK), phospho-specific ERK (p-ERK), phospho-specific RSK-1 (p-RSK-1) and phospho-specific Akt (p-Akt). Equal protein loadings were confirmed using antiactin antibody.

\section{DISCUSSION}

Resorcinol derivatives have been reported to inhibit tyrosinase activity, which is known to regulate the rate-limiting step of melanin synthesis. ${ }^{5)}$ Despite its strong activity, the mechanism of resorcinol-induced hypopigmentation has received little attention. In the present study, we also found that 4-n-butylresorcinol inhibits tyrosinase activity directly. In ad-

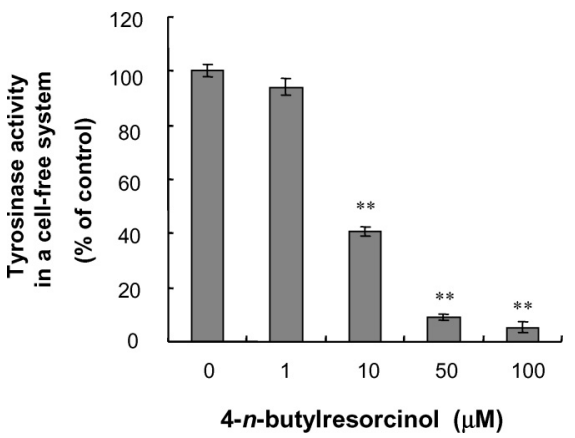

Fig. 5. Effects of 4-n-Butylresorcinol on the Tyrosinase Activity in a CellFree System

To test their direct effects on tyrosinase, human tyrosinase activity was measured in a cell-free system, as described in Materials and Methods. $1-1000 \mu \mathrm{M}$ of 4- $n$-butylresorcinol was added to each well. Results are the averages of triplicate experiments \pm S.D. $* * p<0.01$ compared to control.

dition, 4- $n$-butylresorcinol significantly suppressed melanin synthesis. Therefore, it appears that reduced tyrosinase activity may contribute to the lower pigment contents of 4- $n$ butylresorcinol-treated cells. Since several signaling pathways are involved in the melanogenesis, we investigated the involvements of these mechanisms in 4- $n$-butylresorcinol-induced hypopigmentation.

MITF controls melanocyte pigmentation by regulating the expression of the pigmentation enzyme tyrosinase and tyrosinase-related protein $1{ }^{8,25,26)}$ Several reports have suggested that ERK is an important regulator of melanin synthesis, 


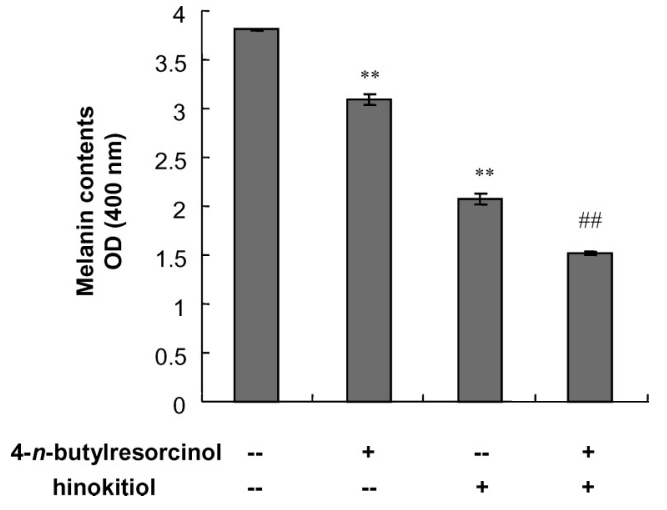

Fig. 6. Effects of 4-n-Butylresorcinol and Hinokitiol on Melanin Synthesis in Mel-Ab Cells

Cells were cultured with $20 \mu_{\mathrm{M}} 4-n$-butylresorcinol with/without $1 \mu \mathrm{M}$ hinokitiol for $4 \mathrm{~d}$, and melanin contents were measured as described in Materials and Methods. Results are the averages of triplicate experiments \pm S.D. $* * p<0.01$ compared to control and \#\# $p<0.01$ compared to the hinokitiol-treated group.

since ERK activation induces MITF phosphorylation and subsequent degradation. ${ }^{17,24,27)}$ A recent study of ours also showed that ERK activation by sphingosine-1-phosphate induces MITF phosphorylation and degradation, and that this results in reduced tyrosinase levels and melanogenesis. ${ }^{15)}$ In addition, we also showed that sphingosine-1-phosphate does not inhibit tyrosinase activity directly. However, in the present study, 4-n-butylresorcinol did not activate the ERK pathway, i.e., MEK, ERK, or RSK-1, and thus 4- $n$-butylresorcinol induced neither MITF phosphorylation nor degradation (Fig. 4). Furthermore, in contrast to sphingosine-1-phosphate, we found that 4- $n$-butylresorcinol inhibits tyrosinase directly (Fig. 5). Therefore, reduced melanin production by 4- $n$-butylresorcinol may be unrelated to the ERK pathway. Concerning the importance of the Akt pathway in melanogenesis, ${ }^{18,28}$ we investigated whether $4-n$-butylresorcinol influences the Akt signaling pathway, but we found that it did not (Fig. 4). Furthermore, 4-n-butylresorcinol had no effect on CREB phosphorylation, which stimulates MITF expression. These results indicate that $4-n$-butylresorcinol may not regulate signaling pathways involved in melanogenesis.

Recently, we reported that hinokitiol reduces MITF and subsequently tyrosinase protein production, which results in a marked reduction in melanin synthesis. ${ }^{29)}$ Thus, the combination of 4- $n$-butylresorcinol and hinokitiol acted in an additive manner, indicating that the strong tyrosinase inhibitory activity of 4- $n$-butylresorcinol works in combination with the reduced level of MITF induced by hinokitiol.

In summary, this study demonstrates that 4- $n$-butylresorcinol does not regulate the ERK, Akt, or CREB pathways. Therefore, we conclude that 4- $n$-butylresorcinol-induced hypopigmentation results from the direct inhibition of tyrosinase.
Acknowledgements This study was supported by a grant (\#02-PJ1-PG1-CH11-0001) from the Good Health R\&D Project, Ministry of Health and Welfare, R. O. K. We also thank Ms. Jin-A Yoon for her excellent technical assistance.

\section{REFERENCES}

1) Kobayashi T., Urabe K., Winder A., Jimenez-Cervantes C., Imokawa G., Brewington T., Solano F., Garcia-Borron J. C., Hearing V. J., ENBO J., 13, 5818-5825 (1994).

2) Yokoyama K., Suzuki H., Yasumoto K., Tomita Y., Shibahara S., Biochim. Biophys. Acta, 1217, 317-321 (1994).

3) Hearing V. J., Jimenez M., Pigment Cell Res., 2, 75-85 (1989).

4) Hearing V. J., Tsukamoto K., FASEB J., 5, 2902-2909 (1991).

5) Tasaka K., Kamei C., Nakano S., Takeuchi Y., Yamato M., Methods Find. Exp. Clin. Pharmacol., 20, 99-109 (1998).

6) Shimizu K., Kondo R., Sakai K., Planta Med., 66, 11-15 (2000).

7) Yasumoto K., Yokoyama K., Takahashi K., Tomita Y., Shibahara S., J. Biol. Chem., 272, 503-509 (1997).

8) Bentley N. J., Eisen T., Goding C. R., Mol. Cell. Biol., 14, 7996-8006 (1994).

9) Bertolotto C., Busca R., Abbe P., Bille K., Aberdam E., Ortonne J. P., Ballotti R., Mol. Cell. Biol., 18, 694-702 (1998).

10) Yavuzer U., Keenan E., Lowings P., Vachtenheim J., Currie G., Goding C. R., Oncogene, 10, 123-134 (1995).

11) Hughes A. E., Newton V. E., Liu X. Z., Read A. P., Nat. Genet., 7, 509-512 (1994).

12) Tassabehji M., Newton V. E., Read A. P., Nat. Genet., 8, 251-255 (1994).

13) Tachibana M., Pigment Cell Res., 10, 25-33 (1997).

14) Englaro W., Bertolotto C., Busca R., Brunet A., Pages G., Ortonne J. P., Ballotti R., J. Biol. Chem., 273, 9966-9970 (1998).

15) Kim D. S., Hwang E. S., Lee J. E., Kim S. Y., Kwon S. B., Park K. C., J. Cell Sci., 116, 1699-1706 (2003).

16) Kim D. S., Kim S. Y., Chung J. H., Kim K. H., Eun H. C., Park K. C., Cell Signal, 14, 779-785 (2002).

17) Wu M., Hemesath T. J., Takemoto C. M., Horstmann M. A., Wells A. G., Price E. R., Fisher D. Z., Fisher D. E., Genes Dev., 14, 301-312 (2000).

18) Khaled M., Larribere L., Bille K., Aberdam E., Ortonne J. P., Ballotti R., Bertolotto C., J. Biol. Chem., 277, 33690-33697 (2002).

19) Price E. R., Horstmann M. A., Wells A. G., Weilbaecher K. N., Takemoto C. M., Landis M. W., Fisher D. E., J. Biol. Chem., 273, 3304233047 (1998).

20) Bertolotto C., Abbe P., Hemesath T. J., Bille K., Fisher D. E., Ortonne J. P., Ballotti R., J. Cell Biol., 142, 827-835 (1998).

21) Dooley T. P., Gadwood R. C., Kilgore K., Thomasco L. M., Skin Pharmacol., 7, 188-200 (1994).

22) Tsuboi T., Kondoh H., Hiratsuka J., Mishima Y., Pigment Cell Res., 11, 275-282 (1998).

23) Busca R., Bertolotto C., Ortonne J. P., Ballotti R., J. Biol. Chem., 271, 31824-31830 (1996).

24) Hemesath T. J., Price E. R., Takemoto C., Badalian T., Fisher D. E., Nature (London), 391, 298-301 (1998).

25) Busca R., Ballotti R., Pigment Cell Res., 13, 60-69 (2000).

26) Tachibana M., Pigment Cell Res., 13, 230-240 (2000).

27) Xu W., Gong L., Haddad M. M., Bischof O., Campisi J., Yeh E. T., Medrano E. E., Exp. Cell Res., 255, 135-143 (2000).

28) Oka M., Nagai H., Ando H., Fukunaga M., Matsumura M., Araki K., Ogawa W., Miki T., Sakaue M., Tsukamoto K., Konishi H., Kikkawa U., Ichihashi M., J. Invest. Dermatol., 115, 699-703 (2000).

29) Kim D. S., Park S. H., Kwon S. B., Li K., Youn S. W., Park K. C., Arch. Pharm. Res., 27, 334-339 (2004). 